\title{
Estudio entomológico de vectores transmisores de la infección por Trypanosoma cruzi en la población rural del estado Sucre, Venezuela
}

\author{
Noris García-Jordán ${ }^{1,2}$, Mariolga Berrizbeitia ${ }^{2,3}$, Juan Luis Concepción ${ }^{4}$, \\ Elis Aldana ${ }^{5}$, Ana Cáceres ${ }^{6}$, Wilfredo Quiñones ${ }^{4}$
}

\footnotetext{
1 Departamento de Biología, Núcleo de Sucre, Universidad de Oriente, Cumaná, Venezuela

2 Posgrado en Biología Aplicada, Núcleo de Sucre, Universidad de Oriente, Cumaná, Venezuela

${ }^{3}$ Instituto de Investigaciones en Biomedicina y Ciencias Aplicadas, Universidad de Oriente, Cumaná, Venezuela

${ }^{4}$ Facultad de Ciencias, Departamento de Biología, Laboratorio de Enzimología de Parásitos, Universidad de Los Andes, Mérida, Venezuela

5 Facultad de Ciencias, Departamento de Biología, Laboratorio de Entomología "Herman Lent", Universidad de Los Andes, Mérida, Venezuela

${ }^{6}$ Unidad de Bioquímica de Parásitos, Centro de Ingeniería Genética, Facultad de Ciencias, Universidad de Los Andes, Mérida, Venezuela
}

Introducción. Debido a los cambios ambientales y a la intervención del hombre en los espacios silvestres, se ha cambiado el nicho ecológico de los vectores reduvídeos.

Objetivo. En el presente estudio se evaluó la situación actual de los índices entomológicos de los triatominos responsables de la transmisión de Trypanosoma cruzi en el estado Sucre.

Materiales y métodos. Se llevó a cabo un estudio prospectivo transversal en 96 centros poblados y 576 viviendas de los 15 municipios del estado Sucre, Venezuela, entre agosto y noviembre de 2008. Los vectores se identificaron con base en sus características morfológicas. La identificación de Trypanosoma spp. en las heces de los triatominos se hizo mediante el examen directo por microscopía. Las láminas positivas se tiñeron con Giemsa y los parásitos se identificaron por sus características morfológicas.

Resultados. Los índices entomológicos con los valores más elevados fueron la dispersión en centros poblados rurales del estado Sucre $(16,67 \%)$ y la colonización de las viviendas $(33,33 \%)$. Las especies de triatominos capturados fueron Rhodnius prolixus, Rhodnius pictipes, Rhodnius robustus, Triatoma maculata y Panstrongylus geniculatus, siendo T. maculata el principal vector en el domicilio.

Conclusiones. A pesar del bajo índice de infección natural en vectores $(1,72 \%)$, la existencia de especies con éxito reproductivo en el domicilio y el peridomicilio puede garantizar el mantenimiento de la cadena epidemiológica, tanto de la enfermedad como del parásito.

Palabras clave: Trypanosoma cruzi, triatominae, zonas rurales, Venezuela.

doi: http://dx.doi.org/10.7705/biomedica.v35i2.2390

\section{Entomological study of Trypanosoma cruzi vectors in the rural communities of Sucre state, Venezuela}

Introduction: The ecological niche of Reduvidae vectors has been modified due to environmental changes and human encroachment into the rural areas.

Objective: This study evaluates the current entomological indices of triatomines responsible for Trypanosoma cruzi infection in Sucre State, Venezuela.

Materials and methods: A cross-sectional and prospective study was conducted in 95 towns and 577 dwellings in the 15 municipalities of the state of Sucre, Venezuela, from August to November, 2008. Triatomine bugs were identified on the basis of morphological characteristics, and their feces examined for T. cruzi infection through direct microscopy. Positive slides were stained with Giemsa and parasites were identified by morphologic characterization.

Results: The entomological indices expressing the highest values were dispersion (16.67\%) and household colonization (33.33\%). The triatomine species captured were: Rhodnius prolixus, Rhodnius

\section{Contribución de los autores:}

Noris García-Jordán: realización de los ensayos, análisis de datos y escritura del manuscrito

Mariolga Berrizbeitia: coordinación del proyecto a nivel regional, colaboración en la escritura del manuscrito y análisis de datos Juan Luis Concepción: coordinación nacional del proyecto, colaboración y revisión del manuscrito

Elis Aldana: asesoramiento en la clasificación taxonómica de los vectores y realización de las pruebas para identificación de especies de triatominos

Ana Cáceres y Wilfredo Quiñones: asesoría en la elaboración del proyecto, y revisión del análisis de datos y del manuscrito 
pictipes, Rhodnius robustus, Panstrongylus geniculatus, and Triatoma maculata, the latter being the main intradomiciliaryvector.

Conclusions: Despite the low index of vector infection (1.72\%), the existence of species with domiciliary and peridomiciliary reproductive success ensures the persistence of the epidemiological chain both for the disease and the parasite.

Key words: Trypanosoma cruzi, triatominae, rural areas, Venezuela. doi: http://dx.doi.org/10.7705/biomedica.v35i2.2390

La enfermedad de Chagas es una infección parasitaria causada por el protozoario flagelado del orden Kinetoplastida, familia Trypanosomatidae, denominado Trypanosoma cruzi (1), la cual puede presentarse en forma aguda, crónica y sistémica. Este protozoario infecta naturalmente a más de 180 especies de mamíferos pertenecientes a los órdenes Didelphimorphia (marsupiales), Lagomorpha (conejos), Chiroptera (murciélagos), Rodentia (roedores), Pilosa (osos perezosos y hormigueros), Cingulata (armadillos), Carnivora (mapache), Primates y Perisodactyla. Infecta también al hombre que, además de padecer la enfermedad, actúa como reservorio (2). En Venezuela, los reservorios silvestres de $T$. cruzi más importantes son los 'rabipelados', armadillos, murciélagos, mapaches, zorros, coatíes, conejos, roedores y monos. Por otro lado, los reservorios domésticos y peridomésticos, son Canis familiaris (perro), Capra hyrcus (cabra), Cavia porcellus (curí), Felis domesticus (gato), Oryctolagus cuniculus (conejo) y Sus scrofa (cerdo), en tanto que los reservorios sinantrópicos son Mus musculus (ratón), Rattus rattus (rata negra) y Rattus norvegicus (rata parda) (3).

Trypanosoma cruzi es transmitido a los mamíferos principalmente por los triatominos, insectos reduvídeos hematófagos de la subfamilia Triatominae, en tres ciclos superpuestos: doméstico, peridoméstico y silvestre (1). Debido a la capacidad que tienen algunas especies de triatominos para habitar en las viviendas humanas y alcanzar grandes densidades de población dentro de ellas, el parásito también circula en los ambientes sinantrópicos (ciclo antropozoonótico, doméstico o secundario), siendo el hombre el principal reservorio doméstico, seguido

\footnotetext{
Correspondencia:

Mariolga Berrizbeitia, Posgrado en Biología Aplicada, Universidad de Oriente, Núcleo de Sucre, Avenida Universidad, Cumaná, estado Sucre, 6101, Venezuela

Teléfono-fax: (58) 2934002270

mberriz@yahoo.com

Recibido: 02/06/14; aceptado: 12/02/15
}

por los perros, gatos y roedores domésticos, lo que permite que también ocurra un ciclo peridoméstico (antropozoonótico o intermediario) (4).

La relevancia de los triatominos en la salud pública radica en su condición de ser los vectores biológicos de la enfermedad de Chagas en ambientes silvestres y sinantrópicos, donde algunas especies transmiten la enfermedad a los humanos o a los animales domésticos; entre ellos se destacan Triatoma infestans, Rhodnius prolixus y Triatoma dimidiata (4). Los triatominos (Hemiptera: Reduviidae, Triatominae) poseen diversos nombres comunes o denominaciones populares en Venezuela, tales como "chipo", "pito", "chupón" y "quipito"(4).

El ciclo de vida y las dinámicas de las poblaciones de las especies de Triatominae, están directamente relacionadas con su interacción con huéspedes vertebrados que les garantizan el suministro continuo de sangre o alimento para desarrollarse y multiplicarse (5). De las 137 especies de triatominos formalmente reconocidas, probablemente todas son capaces de transmitir T. cruzi (6), aunque relativamente pocas tienen relevancia epidemiológica como vectores de $T$. cruzi, entre ellas, $T$. infestans, $R$. prolixus, T. dimidiata, Panstrongylus megistus, Triatoma sordida, Triatoma brasilensis y Rhodnius pallescens (7). Panstrongylus geniculatus, distribuido ampliamente en Suramérica, está asociado principalmente a los ambientes silvestres; sin embargo, en las dos últimas décadas en varios estudios se ha demostrado esta especie en domicilios y su posible participación en epidemias de transmisión oral de T. cruzi (8-11).

En 2000 se reportó en Venezuela un índice de infestación de $R$. prolixus de 5,2 \% (12), en tanto que Triatoma maculata se consideró un vector secundario que se distribuye en el $67 \%$ del territorio nacional y se ha encontrado infectado naturalmente con T. cruzi. Triatoma maculata es una especie adaptada al peridomicilio y al medio selvático (13), sin embargo, se ha reportado su presencia domiciliaria, pues se han detectado huevos y ninfas de estadios II a IV en viviendas del estado Anzoátegui, en el oriente de Venezuela (14). 
En Chile, Uruguay, Argentina y algunas regiones de Brasil, se han implementado programas de control de la enfermedad de Chagas por iniciativa de los países del Cono Sur y el Pacto Andino,,con lo que se ha reducido notablemente la transmisión vectorial (15). En Venezuela, el Programa Nacional de Control de la Enfermedad de Chagas, iniciado en 1950, implementó la estrategia de control vectorial mediante el uso de insecticidas de acción residual (DDT, dieldrín, propoxur, lindano y fenitrotión) en las casas rurales y en su peridomicilio. Posteriormente, se empleó lambdacihalotrina en casas con paredes de bloque de cemento y techos de cinc (16).

En el 2003 se estudió la presencia de vectores en 183 áreas rurales de Venezuela, de las cuales el $28,4 \%$ estaban infestadas con triatominos, con una tasa de infección por $T$. cruzi de 7,8 \% (17). Se encontró 6,5\% de infestación en las viviendas, con tres especies de relevancia para la transmisión de la enfermedad de Chagas: $R$. prolixus, $T$. maculata y $P$. geniculatus, así como un $0,9 \%$ de viviendas infectadas con el parásito (18). En el estado Sucre se han hecho algunos estudios de vectores, sin embargo, no se han reportado los índices entomológicos. En la población indígena Kariña de la parroquia Santa Fe, municipio Sucre, Berrizbeitia, et al. (19), reportaron la presencia de 10 ejemplares del género Panstrongylus sp., de los cuales solo dos (20\%) estaban infectados con Trypanosoma spp. (19). Asimismo, en la población rural de Río Brito (municipio Sucre, estado Sucre), se recolectaron 13 triatominos, todos ellos de la especie $P$. geniculatus, de los cuales $15,38 \%$ resultaron positivos para Trypanosoma spp. (20).

Con la finalidad de obtener datos confiables sobre los vectores de $T$. cruzi en el estado Sucre de Venezuela, se determinaron los índices entomológicos y las principales especies transmisoras de $T$. cruzi en los 15 municipios del estado.

\section{Materiales y métodos}

\section{Área de estudio}

El estudio se realizó de agosto a noviembre de 2008 en el estado Sucre, ubicado en la región nororiental de Venezuela, entre los $10^{\circ} 38^{\prime} 44^{\prime \prime}$ de latitud norte y los $63^{\circ} 2^{\prime} 20^{\prime \prime}$ de longitud oeste. El 60 $\%$ del territorio del estado Sucre está dominado por dos formaciones montañosas, el sistema ArayaParia y el macizo del Turimiquire, cuyas elevaciones máximas alcanzan los 2.600 msnm. La vegetación en el estado incluye biomas del tipo de bosque tropical muy húmedo y del tipo montano bajo, así como bosques secos de tipo tropical, en tanto que en los bosques de las zonas altas se encuentran subpáramos arbustivos (21). El estado Sucre se divide en dos zonas geográficas, la del golfo de Cariaco, conformada por los municipios Montes, Sucre, Cruz Salmerón Acosta, Mejías, Ribero y Bolívar, y la del golfo de Paria, conformada por los municipios Benítez, Andrés Eloy Blanco, Libertador, Bermúdez, Valdez, Andrés Mata, Arismendi, Mariño y Cajigal.

\section{Selección de la muestra}

Se hizo un estudio prospectivo de corte transversal, con un muestreo probabilístico aleatorio y un diseño por conglomerados, en el cual las unidades primarias de muestreo fueron los centros poblados del área rural y, las unidades secundarias, las viviendas. Se seleccionaron de manera aleatoria 96 centros poblados y 576 viviendas (seis viviendas en cada centro poblado), en los 15 municipios del estado Sucre (22).

\section{Recolección de triatominos}

Los insectos se recolectaron mediante capturas activas en el domicilio y en el peridomicilio en las 576 viviendas seleccionadas en cada centro poblado. La inspección de las viviendas y el peridomicilio se hizo después de obtener el consentimiento informado de sus dueños.

El peridomicilio se definió como el área localizada alrededor de la vivienda y delimitada por la cerca perimetral en un área de $20 \mathrm{~m}$ de radio, aproximadamente. Por lo general, en esta área se encuentran depósitos de cosechas, salas de herramientas, depósitos de cosas inservibles, garajes, gallineros, palomares, cochineras y basureros, además de los árboles y palmeras. En las viviendas sin cerca perimetral agrupadas en espacios menores de 50 metros, el peridomicilio se demarcó en la mitad del área entre viviendas contiguas, mientras que en las viviendas aisladas se consideró un área circundante de hasta $50 \mathrm{~m}$.

La revisión de cada vivienda tardaba 30 minutos, durante los cuales una persona hacía la recolección utilizando linternas y pinzas entomológicas (23). Los triatominos recolectados se colocaron en envases identificados con códigos (vivienda, centro poblado y municipio), se taparon con tela de tul y se sujetaron con liga de goma. Los vectores capturados se transportaron al laboratorio para su análisis taxonómico y la detección de Trypanosoma spp. en las heces. 


\section{Identificación taxonómica de los triatominos}

La identificación y la clasificación de los vectores se llevaron a cabo con base en sus características morfológicas; en el caso de los adultos, se examinaron el tamaño, el color, la forma de la banda conexiva, el sexo (por los genitales externos), la forma, el tamaño de la región cefálica y la posición de las antenas, utilizando la clave taxonómica de Lent y Wygodzinsky (24). En el caso de aquellas especies que poseen similitudes morfológicas difíciles de distinguir con base en las características de las claves taxonómicas, se utilizó la metodología descrita por Lent, et al. (25); las ninfas y los estadios de ninfa se identificaron por la presencia de los segmentos del tórax y los vestigios de las alas a partir del cuarto estadio de ninfa (26).

\section{Identificación de Trypanosoma spp. en heces de triatominos}

La extracción de las heces de adultos y ninfas del tercer al quinto estadio en triatominos capturados vivos, se hizo presionando el abdomen sobre una gota de solución salina isotónica en un portaobjeto. En el caso de los ejemplares muertos, adultos y ninfas, se hizo un lavado de la ampolla rectal, en especial, ninfas entre el primer y el segundo estadios, vivas o muertas. Las muestras se fijaron con metanol y, una vez secas, se colorearon con tinción de Giemsa y se observaron en un microscopio óptico con un objetivo de 40X (27).

\section{Análisis de datos}

Los resultados se presentan en porcentajes e índices. Con la información obtenida, se calcularon los índices entomológicos de acuerdo con los criterios establecidos por la Organización Mundial de la Salud (OMS) en el año 2002, con las siguientes fórmulas: infestación de viviendas = número de viviendas con triatominos / número total de viviendas exploradas x 100; infestación del domicilio = número de viviendas con triatominos en el domicilio / número total de viviendas exploradas $x$ 100; infestación del peridomicilio $=$ número de viviendas con triatominos en el peridomicilio / número total de viviendas exploradas x 100; índice de hacinamiento $=$ número total de triatominos capturados / número de viviendas con triatominos; índice de densidad = número total de triatominos capturados / número de viviendas exploradas; dispersión en centros poblados = número de centros poblados con viviendas con triatominos / número de centros poblados explorados x 100; colonización = número de viviendas con ninfas o huevos de triatominos / número de viviendas con triatominos x 100; coinfestación = número de viviendas con más de una especie de triatomino / número de viviendas con triatominos x 100; infección en viviendas = número de viviendas con triatominos infectados con T. cruzi / número de viviendas con triatominos $\times 100$; infección natural (en triatominos) $=$ número de triatominos infectados con $T$. cruzi / número de triatominos capturados x 100 .

\section{Consideraciones éticas}

El protocolo del estudio fue revisado y aprobado por el Comité de Bioética del Bioterio Central de la Universidad de Los Andes (Venezuela).

\section{Resultados}

\section{Estimación de los índices entomológicos de triatominos vectores en el estado Sucre}

De los 15 municipios que conforman el estado Sucre, ocho registraron infestación con vectores $(53,33 \%)$ (figura 1$)$, es decir que en más de la mitad de los municipios había presencia de triatominos vectores. Los municipios con mayor índice de infestación de centros poblados fueron Mariño (60\%) y Benítez y Libertador (33,33 \%). En 21 de las 576 viviendas evaluadas, se encontraron triatominos; las viviendas con mayor índice de infestación por triatominos se ubicaron en las localidades de Punta Brava-La Meseta, San Antonio de Irapa, Soro, Juan Pedro y El Llanito, pertenecientes al municipio de Mariño (18,33 \%), Quebrada de Juan Rojas, del municipio de Libertador (5,55 \%), y Guatamare, El Algarrobo y El Calvario, del municipio de Benítez (5,45 \%) (figura 2).

Los resultados de los índices entomológicos generales se presentan en el cuadro 1 y demuestran que utilizando el método de captura activa, la infestación intradomiciliaria y peridomiciliaria por triatominos en el estado Sucre era baja, si se compara con lo reportado en el estado Lara (municipio de Urdaneta: 80,77 \%), el cual forma parte de los estados del país con mayor endemia para la infección por T. cruzi (28).

El índice de hacinamiento indicó que en cada vivienda infestada con triatominos se encontraron 2,76 ejemplares. El valor de densidad obtenido fue de 0,11 y reveló la presencia de al menos un ejemplar por vivienda. Además, en 16,67 \% de los centros poblados muestreados se encontraron viviendas con triatominos. 


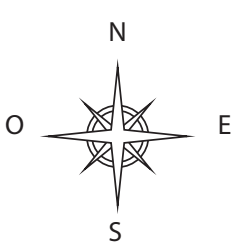

$1: 1137543$
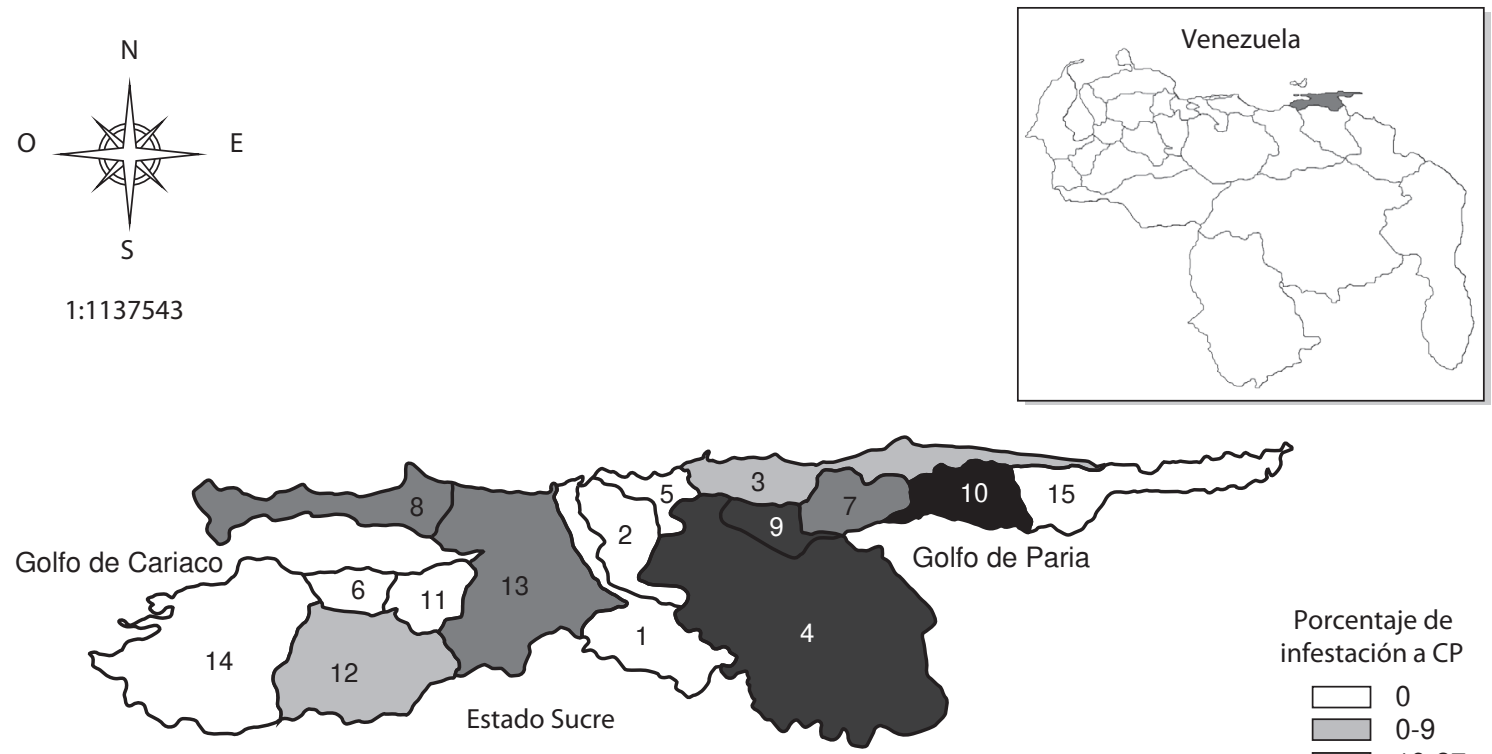

Porcentaje de infestación a CP

1. Andrés Eloy Blanco, 2. Andrés Mata, 3. Arismendi, 4. Benítez, 5. Bermúdez, 6. Bolívar, 7. Cajigal,

8. Cruz Salmerón Acosta, 9. Libertador, 10. Mariño, 11. Mejía, 12. Montes, 13. Ribero, 14. Sucre, 15. Valdez

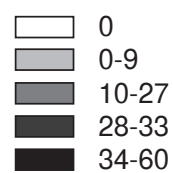

Figura 1. Mapa de los municipios del estado Sucre con infestación de triatominos trasmisores de la infección por Trypanosoma cruzi

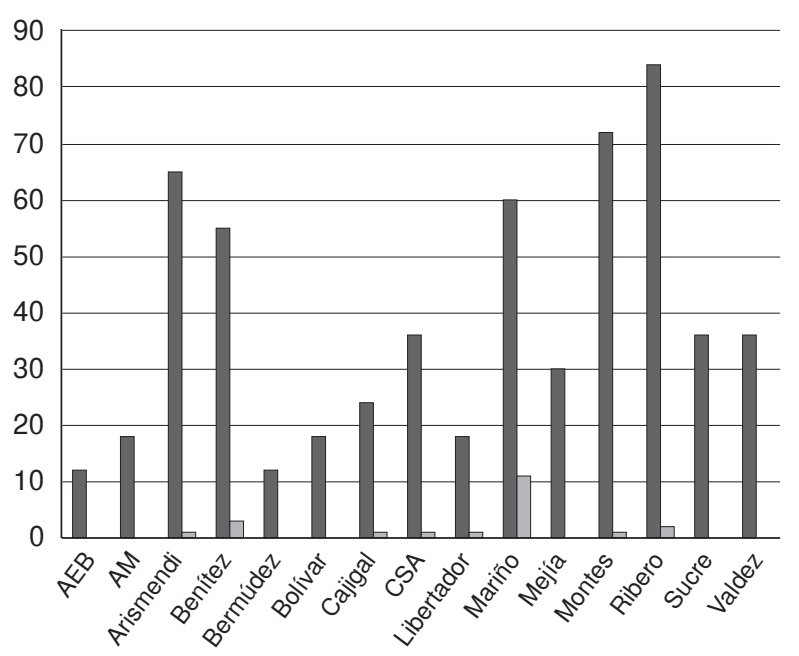

AEB: Andrés Eloy Blanco; AM: Andrés Mata; CSA: Cruz Salmerón Acosta

$\square$ Total de viviendas $\square$ Viviendas infestadas

Figura 2. Número total de viviendas evaluadas e infestadas por triatominos transmisores de la infección por Trypanosoma cruzi en los municipios del estado Sucre, Venezuela

El índice de colonización, que es un indicador del porcentaje de viviendas infestadas con triatominos y evidencia de reproducción del insecto en el domicilio (presencia de ninfas, huevos y exuvias), arrojó un valor de 33,33\%, o sea, aproximadamente tres viviendas colonizadas de cada 10 infestadas, que es un valor alto para este índice.
Igualmente, se encontró un valor relativamente elevado de infestación simultánea con varias especies, revelando que en $9,52 \%$ de las viviendas evaluadas coexistían, al menos, dos especies distintas de triatominos. Sin embargo, la infección natural con triatominos de Trypanosoma spp. fue baja $(1,72 \%)$.

\section{Clasificación taxonómica de las especies de triatominos capturados en el estado Sucre}

El número total de triatominos recolectados fue de 44 adultos y 14 ninfas. El género de triatominos más representado fue Triatoma, con 23 adultos y 11 ninfas, de los cuales 21 ejemplares se identificaron como T. maculata, seguido de Rhodnius con 16 adultos y tres ninfas, siete de los cuales correspondían a $R$. prolixus, uno a $R$. robustus y uno a $R$. pictipes. Además, se capturaron cinco adultos del género Panstrongylus (cuadro 2). Sorpresivamente, la especie que más se encontró infestando el domicilio con adultos fue $T$. maculata, mientras que en el peridomicilio fueron $R$. prolixus, $R$. robustus y $P$. geniculatus. Aunque en pocas viviendas se observó infestación simultánea con varias especies, $R$. prolixus y $P$. geniculatus en el municipio de Benítez, T. maculata y $P$. geniculatus en el municipio de Ribero, y en el municipio de Mariño, en una misma vivienda, se encontraron $R$. prolixus, $T$. maculata y $P$. geniculatus. 
En el cuadro 3, se presenta la distribución de las diferentes especies capturadas en los municipios del estado Sucre, donde el mayor índice de infestación de vectores fue en el municipio de Mariño. Asimismo, se observa que el mayor número

Cuadro 1. Índices entomológicos de los triatominos capturados en el estado Sucre, Venezuela

\begin{tabular}{lc}
\hline $\begin{array}{l}\text { Índices entomológicos en las zonas } \\
\text { rurales del estado Sucre }\end{array}$ & Índice (\%) \\
\hline Infestación de vivienda & $3,65(21 / 576)$ \\
Infestación dentro de la vivienda & $2,95(17 / 576)$ \\
Infestación del peridomicilio & $0,69(4 / 576)$ \\
Hacinamiento* & $2,76(58 / 21)$ \\
Densidad* & $0,10(58 / 576)$ \\
Dispersión de centros poblados & $16,67(16 / 96)$ \\
Colonización & $33,33(7 / 21)$ \\
Infestación con dos o más especies & $9,52(2 / 21)$ \\
Infección en viviendas & $4,76(1 / 21)$ \\
Infección natural triatomínica de T. cruzi & $1,72(1 / 58)$ \\
\hline
\end{tabular}

*Los índices de hacinamiento y densidad no se expresan como porcentajes sino como valores finitos mayores o iguales a 1 , y como símil del concepto de densidad de la Física: masa/volumen, en donde la masa es la cantidad de triatominos capturados y el volumen es el número de viviendas exploradas. de ninfas y adultos fue de T. maculata, tanto en el peridomicilio como en el domicilio. Este resultado evidencia que actualmente, en el estado Sucre, $T$. maculata es la especie más representativa y ha empezado a ubicarse en los domicilios, a pesar de que hasta ahora se había considerado como selvática y no doméstica (29).

En este estudio, la única especie que resultó infectada con Trypansoma spp. fue $R$. prolixus, en un ejemplar capturado en el domicilio de una vivienda ubicada en el centro poblado de El Algarrobo, municipio de Benítez.

\section{Discusión}

Este trabajo es el primer estudio que muestra los índices entomológicos en los 15 municipios del estado Sucre de Venezuela. La recolección activa y sistemática de vectores transmisores de T. cruzi en el domicilio y el peridomicilio, permitió obtener un número total de 58 triatominos en las viviendas estudiadas. El bajo número de vectores recolectados en los 96 centros poblados y 576 viviendas evaluadas, pudo deberse al método de

Cuadro 2. Abundancia de triatominos en función de las especies, el estadio de desarrollo y los biotopos en los 15 municipios del estado Sucre, Venezuela

\begin{tabular}{lcccccc}
\hline \multirow{2}{*}{ Especies } & \multicolumn{3}{c}{ Estadios } & \multicolumn{2}{c}{ Lugar de captura en la vivienda por especies } \\
\cline { 2 - 7 } & Huevos & Ninfas & Adultos & Domicilio (\%) & Peridomicilio (\%) * & Total \\
\hline Rhodnius & 0 & 0 & 10 & $1,74(10 / 576)$ & 0 & 10 \\
Rhodnius prolixus & 0 & 3 & 4 & $1,04(6 / 576)$ & $0,17(1 / 576)$ & 7 \\
Rhodnius robustus & 0 & 0 & 1 & $0,17(1 / 576)$ & 0 & 1 \\
Rhodnius pictipes & 0 & 0 & 1 & 0 & $0,17(1 / 576)$ & 0 \\
Triatoma & 0 & 4 & 2 & $1,04(6 / 576)$ & 0 & 28 \\
Triatoma maculata & 0 & 7 & 21 & $4,86(6 / 576)$ & $0,17(1 / 576)$ & 2 \\
Panstrongylus & 0 & 0 & 2 & $0,17(1 / 576)$ & $0,17(1 / 576)$ & 3 \\
P. geniculatus & 0 & 0 & 3 & $0,35(2 / 576)$ & 4 & 58 \\
Total & 0 & 14 & 44 & 54 & &
\end{tabular}

* porcentaje de triatominos en 576 viviendas

Cuadro 3. Índice de infestación triatomínica de los centros poblados del estado Sucre, Venezuela, discriminados por municipio y especie de triatomino

\begin{tabular}{lccccc}
\hline Municipios & $\begin{array}{c}\text { Índice de } \\
\text { infestación (\%) }\end{array}$ & \multicolumn{3}{c}{ Géneros capturados } \\
\cline { 3 - 6 } & & Rhodnius & Triatoma & Panstrongylus & Total \\
\hline Mariño & 60 & 13 & 11 & 1 & 25 \\
Benítez & 33,33 & 2 & 0 & 2 & 4 \\
Libertador & 33,33 & 1 & 0 & 0 & 1 \\
Cajigal & 25 & 1 & 0 & 0 & 1 \\
Cruz Salmerón Acosta & 16,67 & 1 & 0 & 1 & 24 \\
Ribero & 14,29 & 0 & 0 & 1 & 1 \\
Arismendi & 9,09 & 1 & 0 & 5 & 1 \\
Montes & 8,33 & 0 & 34 & 58 \\
Total & & 19 & & 5 & 5 \\
\hline
\end{tabular}


búsqueda activa, los tiempos de capturas en los muestreos y las condiciones ambientales, ya que las capturas se hicieron durante la estación de lluvias (agosto a noviembre). En tal sentido, se ha clasificado una amplia diversidad de especies de triatominos en biomas con estaciones lluviosas y secas bien definidas, estableciéndose una asociación entre las grandes densidades de población y las estaciones secas prolongadas y con temperaturas altas, hecho que podría explicar la poca cantidad de ejemplares capturados en un tiempo de muestreo caracterizado por la presencia de lluvias en la presente investigación (30).

Otro parámetro que afecta la cantidad de ejemplares en la captura de triatominos, son las horas de búsqueda y recolección. Feliciangeli, et al. (31), demostraron que el método de captura activa de insectos es menos eficaz cuando en la zona de investigación existen limitantes como la hora en que se les captura y plantearon que, posiblemente, se está presentando una nueva situación epidemiológica en Venezuela, en la que aquellos lugares en los cuales se ha interrumpido el ciclo doméstico, la presencia de triatominos en las casas se puede deber al desplazamiento de $R$. prolixus desde las palmas y espacios peridomiciliares hacia el domicilio en horas nocturnas, lo que explica que su presencia sea fácilmente detectada por los residentes de las viviendas durante la noche, mientras que la búsqueda del personal de salud en horas diurnas tiene poco éxito, como ocurrió en la presente investigación.

El índice de infestación de vivienda obtenido fue de $3,65 \%$, inferior a los reportados en los estados Barinas y Lara, de 8 y $15 \%$, respectivamente $(10,32)$. Los resultados obtenidos en este estudio en cuanto a los índices de hacinamiento y de densidad fueron de 2,76 y 0,11 , respectivamente. No se encontraron datos publicados sobre Venezuela con respecto a los índices de hacinamiento y densidad en viviendas. Sin embargo, en un trabajo realizado en 116 viviendas en el municipio de Metztitlán en México, estos índices se emplearon para conocer los riesgos de transmisión de T. cruzi, a pesar de tratarse de diferentes especies de vectores (Triatoma barberi y Triatoma mexicana) y áreas geográficas. En ese trabajo se demostró que $T$. barberi presentaba índices de densidad y hacinamiento de 0,9 y 4,0, respectivamente, duplicando los índices de T. mexicana, lo cual posiblemente favoreció un mayor establecimiento de esa especie en el domicilio (33).
El índice de dispersión de vectores (16,67\%) en los centros poblados evaluados del estado Sucre, es menor al reportado en el estado Lara, donde varió de 80 a $100 \%(28,34)$. Mientras que el índice de colonización (33,33 \%) obtenido en la presente investigación es mayor al reportado por Rodríguez-Bonfante, et al. (13), en el municipio de Andrés Eloy Blanco, estado Lara, en viviendas colonizadas por $P$. geniculatus $(18,18 \%)$ y al reportado por Feliciangeli, et al. (32), quienes determinaron el índice de colonización de $R$. prolixus para el estado Barinas y Portuguesa en 4 y $6 \%$, respectivamente. Sin embargo, se reportó un índice de colonización de 39,19\%, un poco mayor al encontrado en la presente investigación, en el municipio de Urdaneta, estado Lara (34). El éxito de la colonización de los vectores triatominos en áreas endémicas y no endémicas, pone de manifiesto su éxito reproductivo, así como el mantenimiento de la cadena biológica del parásito en biotopos naturales o intervenidos por los humanos (35).

La infestación simultánea describe el porcentaje de viviendas donde se encuentran dos o más especies diferentes de vectores. En este trabajo, se encontró el 9,52\% de las viviendas con infestación simultánea de $T$. maculata, $R$. prolixus y $P$. geniculatus. De forma similar, en diferentes municipios del estado Lara (Urdaneta, Andrés Eloy Blanco, Iribarren), se ha reportado la infestación mixta de las viviendas con $P$. geniculatus y $T$. maculata, en porcentajes que varían entre 1,9 y $10 \%(10,13,34)$. Con respecto a la infestación simultánea de varias especies, Feliciangeli y Torrealba (36) y Zeledón, et al. (37), demostraron que algunas especies selváticas o peridomiciliarias, poco habituales en zonas urbanas o suburbanas, se adaptan a los ambientes domésticos como consecuencia de la perturbación de los ambientes selváticos. Este hecho está estrechamente vinculado con la deforestación y la modificación del hábitat natural, lo que ha llevado a cambios en la disponibilidad de fuentes de alimentación, forzando a las especies a recurrir a fuentes en el domicilio y el peridomicilio humanos (36-38). Es muy probable que, como lo describe Rojas (34) en el estado Sucre, en la parroquia Xaguas, estado Lara, esté ocurriendo una intervención humana en el ambiente que haya resultado en la recolección de especies silvestres como $T$. maculata y $P$. geniculatus en el domicilio.

Es probable que los mayores índices de infestación triatomínica encontrados en la región del golfo de Paria, se hayan debido a una mayor 
intervención del hombre en los espacios silvestres de esa región y a un mayor control vectorial en el pasado mediante la aplicación de insecticidas en los municipios del golfo de Cariaco, identificados por el Programa Nacional de Control de la Enfermedad de Chagas como aquellos con las mayores tasas de infección por $T$. cruzi en humanos. Probablemente, las características bioclimáticas del golfo de Cariaco: temperaturas que oscilan entre los 19 y $27{ }^{\circ} \mathrm{C}$; humedad entre 70 (época seca) y $80 \%$ (época húmeda); clima semiárido; pluviosidad anual de $375 \mathrm{~mm}$, y altitud entre los 3 y los 2.600 msnm, así como contar con algunos municipios caracterizados por ser zonas de piedemonte y agrícolas (Sucre, Mejías, Bolívar y Montes), expliquen la mayor población de triatominos en esa área geográfica (39).

El golfo de Paria tiene una temperatura entre 25 y $34{ }^{\circ} \mathrm{C}$, una humedad relativa que varía entre $80 \%$ en época seca y $90 \%$ en época húmeda, una altitud entre 200 y $800 \mathrm{msnm}$, una pluviosidad anual entre 1.200 y $2.000 \mathrm{~mm}$, y un clima típico de regiones boscosas. Algunos de sus municipios, donde no se encontró infestación de triatominos (Andrés Mata), se caracterizan por ser zonas de llanura con pocas extensiones boscosas, en tanto que los que presentaron el mayor índice de infestación de centros poblados, se caracterizan por ser zonas de piedemonte (Libertador, Benítez y Cajigal). En el caso del municipio de Mariño, este se encuentra dividido geográficamente en dos áreas: la zona norte se asemeja a un bosque de montaña o piedemonte, y la zona sur es una planicie con matorrales que llega hasta las costas del océano Atlántico (39).

El estudio entomológico reveló que solo uno $(1,72 \%)$ de los triatominos estaba infectado por Trypanosoma spp. Este bajo índice de infección pudo deberse al hecho de que no se empleó el diagnóstico molecular en el caso de los vectores que se encontraban muertos, ya que en ellos parte del contenido estomacal se había perdido en el proceso de la muerte del ejemplar. Esto dificultó el análisis de las heces en intestino y ampolla rectal, para verificar la presencia de $T$. cruzi.

Se han reportado bajos índices de infección vectorial por T. cruzi en el estado Portuguesa (1,09\%), en poblaciones del estado Lara $(0,004-0,36 \%)$ y en las poblaciones de Cumboto y Periquito del estado Aragua $(0 \%)(28,32,40)$. Sin embargo, existen otros autores que reportan un alto índice de infección vectorial en zonas endémicas del país (41).
En este estudio también se identificaron las especies que habitaban tanto en el domicilio como en el peridomicilio. La especie que más se encontró infestando el domicilio fue $T$. maculata (ejemplares adultos), mientras que en el peridomicilio fueron adultos de las especies $R$. prolixus, $R$. robustus y $P$. geniculatus. Asimismo, en la presente investigación se demostró que $T$. maculata es el principal vector en el estado Sucre y que se ha habituado en las viviendas rurales evaluadas, ya que en estas se encontraron adultosy ninfas. La ocupación del domicilio por especies silvestres, como se ha demostrado en diversos trabajos $(13,34)$ y en la presente investigación, pone en evidencia que especies como T. maculata, tradicionalmente consideradas vectores secundarios por encontrárselas en ambientes de menor riesgo epidemiológico como el peridomicilio, podrían ahora considerarse de mayor riesgo epidemiológico al demostrarse su colonización del ambiente domiciliario. En Venezuela el principal transmisor de la enfermedad de Chagas es $R$. prolixus, cuyo hábitat es predominantemente domiciliario (42). Sin embargo, en los estudios de Feliciangeli, etal. (36), y de Reyes-Lugo y Rodríguez, et al. (11), se ha reportado que en los últimos años la invasión del hombre en zonas silvestres y el crecimiento urbano cercano al hábitat natural de los triatominos, han propiciado el incremento en el domicilio de $T$. maculata y $P$. geniculatus, cuyos hábitats tradicionales son el peridomicilio y las áreas silvestres, respectivamente. Esto sugiere que $R$. prolixus puede estar siendo desplazado por estas especies silvestres en el domicilio.

Un trabajo reciente estableció el desplazamiento de T. maculata al hábitat doméstico mediante estudios morfométricos y de genotipificación que evaluaron la variabilidad de las alas y el polimorfismo de sus marcadores genotípicos (mtCyt b y la región del gen tubulina beta) en subpoblaciones de diferentes hábitats en Venezuela (Anzoátegui, Bolívar, Portuguesa, Monagas, Nueva Esparta y Sucre) (43). En ese trabajo se comprobó la adaptación domiciliaria de T. maculata, ya que se demostró la discriminación que esta especie hace de sus biotopos. Los especímenes selváticos y peridomésticos fueron más similares entre ellos en cuanto a la arquitectura de sus alas y a la variabilidad del marcador tubulina beta, en comparación con los triatominos capturados en el biotopo domiciliar. Sin embargo, la amplificación del gen $m t C y t b$ presentó poca variabilidad entre $T$. maculata de diferentes áreas geográficas y biotopos (43). Igualmente, Torres, et al. (44), reportaron diferencias en la 
conformación de las alas y la cabeza de $T$. maculata en diferentes biotopos (domicilio y peridomicilio), lo cual sugería que en el municipio de Xaguas (Venezuela) esta especie podría estar adaptándose al domicilio con fuentes sanguíneas diferentes al humano y comenzando a adaptarse, asimismo, a la sangre humana como fuente de alimentación. La colonización domiciliaria de esta especie podría estar asociada a variables del medio ambiente que facilitan su penetración en las viviendas humanas, como la presencia de palmas de coco en el ambiente peridomiciliar, las cuales constituyen un nicho ecológico apropiado para $T$. maculata y $R$. prolixus (45). Asimismo, la asociación de $T$. maculata con el reptil Thecadactylus rapicauda es una estrategia efectiva para la colonización de viviendas y este hecho podría tener implicaciones de gran importancia para la transmisión domiciliaria de la infección por $T$. cruzi (46).

Los resultados de la presente investigación demuestran que $T$. maculata está colonizando viviendas rurales del estado Sucre. Varios investigadores han reportado resultados similares a los presentados en este estudio, en el cual se demuestra la colonización domiciliaria de T. maculata $(13,28,34,44)$. En Venezuela se han descrito el tipo de viviendas y los alimentos preferidos por $T$. maculata en áreas endémicas: es primordialmente un ornitófago y se la encuentra en el peridomicilio (38).También, en este estudio se demuestra la presencia de las especies $R$. robustus en el domicilio y $R$. pictipes en el peridomicilio. Estas especies de Rhodnius han sido reportadas tanto en áreas endémicas como no endémicas, destacándose su presencia en palmares, bromelias y cuevas en ambientes silvestres $(44,47)$. Es importante destacar que $P$. geniculatus y $R$. robustus se encontraron dentro de las viviendas analizadas, a pesar de caracterizarse por ser selváticas,y que varios autores han descrito a $R$. robustus como una especie de triatomino que mantiene el ciclo silvestre de $T$. cruzi, la cual está estrechamente relacionada con $R$. prolixus. Por otra parte, aunque $R$. pictipes es una especie a la que no se le conocen comportamientos antropofílicos, su papel como vector de la enfermedad de Chagas no debe subestimarse (24).

El aspecto más relevante de la presente investi-gación radica en que se se hicieron búsquedas activas de vectores transmisores de la infección por T. cruzi en 576 viviendas y 96 centros poblados de los 15 municipios que conforman el estado Sucre, y se demostró que T. maculata es el principal vector en el estado, habiéndose adaptado a las viviendas en las poblaciones rurales. La existencia en el domicilio de especies con éxito reproductivo y hábitos alimenticios asociados a animales domésticos y a humanos, podría implicar que se mantenga la cadena epidemiológica domiciliar de la transmisión vectorial de la enfermedad de Chagas.

\section{Conflicto de intereses}

Los autores declaran no tener conflicto de intereses en relación con este estudio.

\section{Financiación}

Este proyecto fue financiado por el Ministerio del Poder Popular para la Salud, Dirección de Investigación y Educación, Proyecto $\mathrm{N}^{\circ}$ 490029000 .

\section{Referencias}

1. Rassi A, Anis RJ, Marín-Neto JA. Chagas disease. Lancet. 2010;375:1388-402. http://dx.doi.org/10.1016/S0140-6736 (10)60061-X.

2. Herrera L. Una revisión sobre reservorios de Trypanosoma (Schizotrypanum) cruzi (Chagas, 1909), agente etiológico de la enfermedad de Chagas. Bol Mal Salud Amb. 2010; 50:3-15.

3. Organización Mundial de la Salud. Control of Chagas disease. Fecha de consulta: 15 marzo de 2012. Disponible en: http://whqlibdoc.who.int/trs/ WHO_TRS_905.pdf.

4. Cazorla D, Nieves E. Triatominae de Venezuela: distribución geográfica, aspectos taxonómicos, biológicos e importancia médica. Avances Cardiológicos. 2010;30:347-69.

5. Souza R de C, Soares AC, Alves CL, Lorosa ES, Pereira MH, Diotaiuti L. Feeding behavior of Triatoma vitticeps (Reduviidae: Triatominae) in the state of Minas Gerais, Brazil. Mem Inst Oswaldo Cruz. 2011;106:16-22. http://dx. doi.org/10.1590/S0074-02762011000100003

6. Coura JR. Chagas disease: What is known and what is needed--a background article. Mem Inst Oswaldo Cruz. 2007;102:113-22. http://dx.doi.org/10.1590/S007402762007000900018

7. Schofield CJ, Galvao C. Classification, evolution, and species groups within the Triatominae. Acta Trop. 2009;110:88-100.

8. Alarcón de Noya B, Díaz-Bello Z, Colmenares C, RuizGuevara R, Mauriello L, Zavala-Jaspe R, et al. Large urban outbreak of orally acquired acute Chagas disease at a school in Caracas, Venezuela. J Infect Dis. 2010;201:130815. http://dx.doi.org/10.1086/651608

9. Carrasco HJ, Torrellas A, García C, Segovia M, Feliciangeli MD. Risk of Trypanosoma cruzi I (Kinetoplastida: Trypanosomatidae) transmission by Panstrongylus geniculatus (Hemiptera: Reduviidae) in Caracas (Metropolitan District) and neighboring States, Venezuela. Int J Parasitol. 2005;35:1379-84. http://dx.doi. org/10.1016/j.ijpara.2005.05.003 
10. Feliciangeli MD, Carrasco H, Patterson JS, Suárez B, Martínez C, Medina M. Mixed domestic infestation by Rhodnius prolixus Stal, 1859 and Panstrongylus geniculatus Latreille, 1811, vector incrimination, and seroprevalence for Trypanosoma cruzi among inhabitants in El Guamito, Lara State, Venezuela. Am J Trop Med Hyg. 2004;71:501-5.

11. Reyes-Lugo M, Rodríguez-Acosta A. Domiciliation of the sylvatic Chagas disease vector Panstrongylus geniculatus Latreille, 1811 (Triatominae: Reduviidae) in Venezuela. Trans R Soc Trop Med Hyg. 2000;94:508.

12. Organización Panamericana de la Salud. Iniciativa de los países de Centro América para la interrupción de la transmisión vectorial y transfusional de la enfermedad de Chagas. Reunión internacional para el establecimiento de criterios de certificación de la eliminación de Rhodnius prolixus (Stal, 1859).Fecha de consulta: 15 de marzo de 2012. Disponible en: http://www1.paho.org/spanish/ad/dpc/ cd/dch-ca.htm

13. Rodríguez-Bonfante C, Amaro A, García M, Mejías LE, Guillén P, García R, et al. Epidemiology of Chagas disease in Andrés Eloy Blanco, Lara, Venezuela: Triatomine infestation and human seroprevalence. Cad Saúde Pública. 2007;23:1133-40. http://dx.doi.org/10.1590/S0102-311X 2007000500015

14. Morocoima A, Sotillo E, Salaverría C, Maniscalchi M, Pacheco F, Chique D. Domiciliación del vector peridomiciliario de la enfermedad de Chagas, Triatoma maculata (Ericsson 1848) en caserío rural del norte del estado Anzoátegui. Acta Cient Venez. 2004;55:215.

15. Moncayo A. Chagas disease: Current epidemiological trends after the interruption of vectorial and transfusional transmission in the Southern Cone countries. Mem Inst Oswaldo Cruz. 2003;98:577-91. http://dx.doi.org/10.1590/ S0074-02762003000500001

16. Ache A, Matos AJ. Interrupting Chagas disease transmission in Venezuela. Rev Inst Med Trop Sao Paulo. 2001;43:37-43. http://dx.doi.org/org/10.1590/S0036-46652001000100008

17. Organización Panamericana de la Salud. Tripanosomiasis americana (enfermedad de Chagas). Bol Epidemiol. 2003;24:15-6.

18. Organización Panamericana de la Salud. Informe final: Reunión Internacional para el Establecimiento de Criterios de Certificación de la Eliminación de Rhodnius prolixus. Fecha de consulta: 15 de abril de 2012. Disponible en: http://www.paho.org/Spanish/AD/DPC/CD/dch-ca.htm.

19. Berrizbeitia M, Moreno D, Ward BJ, Gómez E, Jorquera A, Rodríguez J, et al. Trypanosoma cruzi Infection in an Indigenous Kariña Community in Eastern Venezuela. Epidemiol Res Int. 2012;2012:7. http://doi.dx. org/10.1155/2012/138259

20. Berrizbeitia M, Ward BJ, Bubis J, Gottschalk M, Ache A, Perdomo D, et al. 85-kDa protein of Trypanosoma cruzi purified by affinity chromatography used in the multiple antigen binding assay (MABA) for the diagnosis of $T$. cruzi infection in a Venezuelan rural community.Parasitol Res. 2010;106:1127-34. http://doi.dx.org/10.1007/s00436-0101773-6

21. Instituto Nacional de Estadística. Informe geoambiental estado Sucre. 2011. Fecha de consulta: 17 de diciembre de 2014. Disponible en: http://www.academia.edu/9041667/ Informe_GeoambientaLSSucre
22. Berrizbeitia M, Concepción J, Carzola V, Rodríguez J, Cáceres A, Quiñones W. Seroprevalencia de la infección por Trypanosoma cruzi en Canis familiaris del estado Sucre, Venezuela. Biomédica. 2013;33:214-25. http://dx. doi.org/10.7705/biomedica.v33i2.760

23. Schofield CJ. A comparison of sampling techniques for domestic populations of triatominae.Trans R Soc Trop Med Hyg. 1978;72:449-55.

24. Lent $\mathbf{H}$, Wygodzinsky $\mathbf{P}$. Revision of the triatominae (Hemiptera, Reduviidae) and their significance as vectors of Chagas' disease. Bull Amer Mus Nat His. 1979;163:125520 .

25. Lent H, Jurberg J, Galvão C. Rhodnius stali n. sp. related to Rhodnius pictipes Stal, 1872 (Hemiptera, Reduviidae, Triatominae). Mem Inst Oswaldo Cruz. 1993;88:605-614. http://dx.doi.org/10.1590/S0074-02761993000400019

26. Carcavallo R, Galíndez-Girón I, Jurberg J, Galvao C, Lent H. Pictorial keys for tribes, genera and species of the subfamily triatominae. In: Carcavall RU, Galíndez-Girón I, Jurberg J, Lent $\mathrm{H}$, editors. Atlas of Chagas disease vectors in the Americas. Río de Janeiro: Fiocruz; 1999. p.107-244.

27. Silveira A, Sanches O. Guía para muestreo de actividades de vigilancia y control vectorial de la enfermedad de Chagas. Fecha de consulta: 11 de mayo de 2011. Disponible en: http://www.bvsops.org.uy/pdf/chagas09.pdf.

28. Bonfante-Cabarcas R, Rodríguez-Bonfante C, Vielma BO, García D, Saldivia AM, Aldana E, et al. Seroprevalence for Trypanosoma cruzi infection and associated factors in an endemic area of Venezuela. Cad Saúde Pública. 2011;27:1917-29. http://dx.doi.org/10.1590/S0102-311X 2011001000005

29. Guhl F. La iniciativa andina para el control de la enfermedad de Chagas: situación epidemiológica y acciones de control en la región colombo - venezolana. Acta Biol Venez. 2006;26:23-34.

30. de Casas S, Carcavallo R, Galíndez I, Burgos J. Bioclimatic factors and zones of life. In: CarcavalloRU, Galíndez-Girónl, Jurberg $\mathrm{J}$, Lent $\mathrm{H}$, editors. Atlas of Chagas disease vectors in the Americas. Río de Janeiro: Fiocruz; 1999. p. 747-92.

31. Feliciangeli $D$, Hernández $M$, Suárez $B$, Martínez $C$, Bravo A, Bracho J, et al. Comparación de métodos de captura intradoméstica de triatominos vectores de la enfermedad de Chagas en Venezuela. Bol Mal Salud Amb. 2007:47:103-17.

32. Feliciangeli MD, Sánchez-Martín MJ, Suárez B, Marrero R, Torrellas A, Bravo A, et al. Risk factors for Trypanosoma cruzi human infection in Barinas State, Venezuela. Am J Trop Med Hyg. 2007;76:915-21.

33. Becerril M, Ángeles V, Noguez J, Imbert J. Riesgo de transmisión de Trypanosoma cruzi en el municipio de Metztitlán, estado de Hidalgo, México, mediante la caracterización de unidades domiciliares y sus índices entomológicos. Neotrop Entomol. 2010;39:810-17. http://dx. doi.org/10.1590/S1519-566X2010000500021

34. Rojas ME, Varquez P, Villarreal MF, Velandia C, Vergara $\mathrm{L}$, Morán-Borges $\mathrm{YH}$, et al. An entomological and seroepidemiological study of Chagas' disease in an area in central-western Venezuela infested with Triatoma maculata (Erichson 1848). Cad Saúde Pública. 2008;24:2323-33. http://dx.doi.org/10.1590/S1519-566X2010000500021 
35. Gómez A, Grant M, Hernández N, Hurtado Y, Sivira C, Zambrano G. Seroprevalencia de la enfermedad de Chagas en humanos y caninos y factores de riesgo. Areas de influencia, Ambulatorio San Pedro de Monserrat y San Miguel, Parroquia San Miguel, Municipio Urdaneta, estado Lara. [Tesis]. Barquisimeto: Universidad Centro Occidental Lisandro Alvarado; 2005.

36. Feliciangeli D, Torrealba J. Observaciones sobre Rhodnius prolixus (Hemiptera, Reduviidae) en su biotopo silvestre Copernicia tectorum. Bol Dir Malariol San Amb. 1977;17:198-205.

37. Zeledón R, Solano G, Burstin L, Swartzwelder JC. Epidemiological pattern of Chagas' disease in an endemic area of Costa Rica.Am J Trop Med Hyg. 1975;24:214-25.

38. Pifano F. La epidemiología de la enfermedad de Chagas en Venezuela. Arch Venez Med Trop Parasitol Med. 1973;5:171

39. Villa M. Aspectos geográficos del estado Sucre. Serie Monografías estadales. Caracas: Coorporación Venezolana de Fomento; 1965. p. 1-266.

40. Serrano O, Mendoza F, Suárez B, Soto A. Seroepidemiología de la enfermedad de Chagas en dos localidades del municipio Costa de Oro, estado Aragua, Venezuela. Biomédica. 2008;28:108-15. http://dx.doi. org/10.7705/biomedica.v28i1.113

41. González-Britez N, Morocoima A, Martínez C. Infección por Trypanosoma cruzi y polimorfismo del citocromo B del ADN mitocondrial en Triatoma maculata de Anzoategui y Portuguesa, Venezuela. Bol Mal Salud Amb. 2010;50:85-93.

42. Feliciangeli MD, Campbell-Lendrum D, Martínez C, González D, Coleman P, Davies C. Chagas disease control in Venezuela: Lessons for the Andean region and beyond. Trends Parasitol. 2003;19:44-9. http://dx.doi.org/10.1016/ S1471-4922(02)00013-2

43. García-Alzate R, Lozano-Arias D, Reyes-Lugo RM, Morocoima A, Herrera L, Mendoza-León A. Triatoma maculata, the vector of Trypanosoma cruzi, in Venezuela. Phenotypic and genotypic variability as potential indicator of vector displacement into the domestic habitat. Front Public Health. 2014;2:1-9. http://dx.doi.org/10.3389/fpubh. 2014.00170

44. Torres K, Avendaño-Rangel F, Lizano E, Rojas M, Rodríguez-Bonfante $\mathrm{C}$, Bonfante-Cabarcas $\mathrm{R}$, et al. Evaluación de la estructura espacial de Triatoma maculata del centro-occidente de Venezuela y su viabilidad alimentado con sangre humana en condiciones de laboratorio. Biomédica. 2010;30:72-81. http://dx.doi.org/10.7705/ biomedica.v30i1.155

45. Morocoima A, Chique J, Zavala-Jaspe R, Díaz-Bello Z, Ferrer E, Urdaneta-Morales S, et al. Commercial coconut palm as an ecotope of Chagas disease vectors in northeastern Venezuela. J Vector Borne Dis. 2010;47:76-84.

46. Reyes-Lugo M, Reyes-Contreras M, Salvi I, Gelves W, Avilán A, Llavaneras D, et al. The association of Triatoma maculata (Ericsson 1848) with the gecko Thecadactylus rapicauda (Houttuyn 1782) (Reptilia: Squamata: Gekkonidae): A strategy of domiciliation of the Chagas disease peridomestic vector in Venezuela? Asian Pac J Trop Biomed. 2011;1:279-84. http://dx.doi.org/10.1016/S2221-1691(11) 60043-9

47. Guerrero L, Scorza JV. Las fuentes alimenticias de algunos Triatominae silvestres en los llanos centro-occidentales de Venezuela. Bol Dir Mal San Amb. 1981;21:129-39. 